\title{
Anomalous role of information diffusion in epidemic spreading
}

\author{
Xiaochen Wang $\odot,{ }^{1}$ Xuzhen Zhu, ${ }^{2}$ Xiaofeng Tao, ${ }^{1}$ Jinghua Xiao, ${ }^{3,4}$ Wei Wang $\odot,{ }^{5, *}$ and Ying-Cheng Lai $\odot^{6}$ \\ ${ }^{1}$ National Engineering Laboratory for Mobile Network Technologies, Beijing University of Posts and Telecommunications, \\ Beijing 100876, China \\ ${ }^{2}$ State Key Laboratory of Networking and Switching Technology, Beijing University of Posts and Telecommunications, Beijing 100876, China \\ ${ }^{3}$ School of Science, Beijing University of Posts and Telecommunications, \\ Beijing 100876, China \\ ${ }^{4}$ State Key Laboratory of Information Photonics and Optical Communications, Beijing University of Posts and Telecommunications, Beijing \\ 100876, China \\ ${ }^{5}$ Cybersecurity Research Institute, Sichuan University, Chengdu 610065, China \\ ${ }^{6}$ School of Electrical, Computer and Energy Engineering, Arizona State University, Tempe, Arizona 85287, USA
}

(Received 27 July 2020; accepted 4 February 2021; published 17 February 2021)

\begin{abstract}
A widely held belief in network epidemiology is that information diffusion makes individuals aware of the epidemic and thus drives them to seek protections from nonpharmaceutical or pharmaceutical resources, which can help suppress its spread. However, as the COVID-19 pandemic has revealed, excessive information diffusion can trigger irrational acquisition and hoarding behaviors, which can lead to shortages of resources even for those in urgent need, consequently, worsening disease spreading. To develop a quantitative understanding of the effect of information diffusion on epidemic spreading, subject to allocations of limited resources, has become an urgently important problem with broad implications. We construct a multiplex network framework to characterize the complex interplay among resource allocation, information diffusion, and epidemic spreading, and develop a microscopic Markov chain theory to analyze their coevolution dynamics. There are two main findings. Firstly, if infected individuals have a large recovery probability, information diffusion plays the expected "normal" role of suppressing the epidemic. However, if the recovery probability is low, information diffusion can anomalously worsen the spread, regardless of the available resources insofar as they are limited. Secondly, different types of resources can lead to distinct phase transitions underlying the epidemic outbreak when the recovery probability is low: with limited cure-focused resources, the phase transition is of the second order, but if resources are of the protection type, the transition becomes first order, and a hysteresis loop emerges. The generality of the findings is established through simulations of synthetic and empirical three-layer networks with results in agreement with the theoretical predictions.
\end{abstract}

DOI: 10.1103/PhysRevResearch.3.013157

\section{INTRODUCTION}

In an epidemic or global pandemic, such as SARS [1], H1N1 influenza [2], and the ongoing novel coronavirus (COVID-19) pandemic [3,4], the availability and distribution of different types of resources can play an important role in suppressing the spread. There are two main categories of resources: public and individual. Public resources are accessible to all individuals in society, and they include hospitals, government testing and monitoring facilities, and different levels of disease control agencies. Individual-based resources, by definition, depend on the individual and are typically not equally distributed in society. The possession of resources

\footnotetext{
*wwzqbx@hotmail.com

Published by the American Physical Society under the terms of the Creative Commons Attribution 4.0 International license. Further distribution of this work must maintain attribution to the author(s) and the published article's title, journal citation, and DOI.
}

is largely determined by information spreading. In a sudden outbreak, individuals who are fully aware of the epidemic at the earliest stage tend to pursue and secure more resources required for preventing infection than those who are unaware of the disease. In the real world, available medical resources, whether protection or cure focused, are limited. The diffusion of disease-related information may cause a public panic and induce depletion of the limited resources in an undesired and even harmful manner. For example, in the COVID-19 pandemic, information has spread extremely rapidly around the globe, sending susceptible individuals to obtain personal protective equipment in an irrational manner. In the early stage, there was a significant shortage of face masks in many countries for most individuals except for a few with a high level of awareness who secured a sufficient supply. The fear created by the pandemic stimulated irrational behaviors such as "panic buying" and hoarding [5]. As a result, in many places, resources were depleted, causing severe shortages even for frontline health workers and patients who needed resources most [6]. Situations can thus arise where information diffusion would likely worsen the epidemic by depriving certain 
individuals of resources and making them more vulnerable to infection. Because of the supply shortage, public distribution of resources to society in an equal and fair way becomes unlikely or even impossible. Instead, individuals rely on information sharing and social connections to obtain a share of the resources. To understand the effects of resource and information sharing on epidemic spreading at the individual level is of paramount importance. Because of the lack of empirical data associated with such effects, a viable way at present is to resort to mathematical modeling, which has been well justified as an effective epidemic forecasting tool $[7,8]$. In spite of the large body of literature on network epidemic modeling, to our knowledge, a framework incorporating both resource distribution and information diffusion is not available. More specifically, how information diffusion interacts with resource distribution to affect epidemic spreading in terms of the incidence, outbreak, and phase transition and whether or not an optimal strategy can be devised for resource usage have become outstanding questions. A good understanding of how the responses or behaviors of individuals affect the spreading dynamics can facilitate effective government policymaking in terms of resource allocation [9], controlled management of public behaviors, and information dispensing [10-12] to contain epidemic spreading.

To place our contribution in the proper context, we present a brief survey of what is known about the effects of information and resource sharing on epidemic spreading. In the modern era, when an outbreak occurs, information about the epidemic diffuses rapidly through online social media and digital communication channels, which can profoundly change individual behaviors and steer the dynamical process of spreading, for better or worse. For example, infected individuals would inform their friends about the disease, spreading disease-related information to various social networks [13]. Once an individual is aware of the disease, to prevent from being infected, he or she may take some protective measures by exploiting medical resources or changing social behaviors such as practicing social distancing or undergoing home quarantine, which can alter the dynamical process of epidemic spreading [14-17]. There have been previous studies on the interacting contagion dynamics between the epidemic and awareness. For example, if the population is well mixed, a result [18] is that the disease-related information diffusion can reduce the epidemic size but leaves the epidemic threshold unchanged. As the social exchange of information occured in cyberspace and the actual disease spreading took place in the physical space, multiplex networks were introduced $[13,19,20]$ to study the coupled dynamical processes of epidemic spreading and information diffusion, with the general finding that information exchange can have dramatic effects on the epidemic outbreak. The influence of diffusion characteristics was also studied [21,22]. More recently, the effect of the emotional tendency $[10,23]$ on spreading dynamics has been investigated.

Resources play the uttermost important role in suppressing epidemic spreading; the containment of an epidemic cannot be achieved without the necessary health resources [24]. Generally, infected individuals use various medical resources, but the healthy population is responsible for the reproduction of resources. From a modeling perspective, the consumption and regeneration of resources can be incorporated into the classical susceptible-infected-susceptible (SIS) framework. One result was that an epidemic may go out of control and be explosive when the cost of recovery of sick individuals is above a critical value [25]. The role of investment of funds in making the public aware [26] and the usage strategy of medical resources in epidemic spreading were also studied [27,28]. Public resources notwithstanding, a common scenario is that individuals gain support and resources through their social networks from family members and friends [29,30]. In this regard, a previous paper [31] explored how resource allocation among neighbors in a social network and the adaptive evolution of the network topology [32] affect the epidemic outbreak.

In this paper, we articulate a multiplex network model to investigate the coevolution dynamics of information diffusion, resource allocation, and epidemic spreading. To characterize the resource efficacy, we designate a parameter in the unit interval, whose ranges of variation naturally divide the resources into two kinds: protection and cure focused. Employing the microscopic Markov chain (MMC) theory, we predict the existence of an optimal type of resources to contain epidemic spreading, depending on the circumstances. In particular, we identify two distinct cases: infected individuals have a relatively large or small recovery probability. In the former case, protection-focused resources are more effective at suppressing the epidemic, but for the latter, cure-focused resources are more helpful. Depending on the recovery probability, information diffusion can play opposite roles: it can suppress spreading if this probability is large, but it can anomalously worsen the epidemic in the case of a small recovery probability. For the latter case, the nature of the phase transition underlying the epidemic outbreak can be characteristically distinct: a continuous, second-order type when allocated resources are cure focused and a discontinuous, first-order type with the emergence of a hysteresis loop when resources are protection focused. These results provide insights into the anomalous effect of information diffusion in the early phase of COVID-19. Our findings suggest, as a quantitative level, the uttermost importance of enhancing the recovery rate in suppressing the epidemic.

\section{INFORMATION-RESOURCE-EPIDEMIC COEVOLUTION MODEL}

Figure 1(a) illustrates, qualitatively, the interplay among information diffusion, resource allocation, and epidemic spreading. The emergence of an epidemic in a physical contact network triggers the diffusion of pertinent information in the corresponding online-offline social network, and the dissemination of information drives aware individuals to seek resource support.

During the outbreak of an epidemic, resources are always limited and generated by the healthy population as infected individuals have lost their productivity. Healthy individuals, thus, have more advantages by securing resource support and bear the cost to benefit others [5]. In this case, individuals are more likely to and it is also convenient for them to gain support through their social networks from family members and friends [29]. (Our paper focuses on this situation 


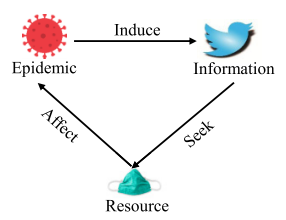

(b)

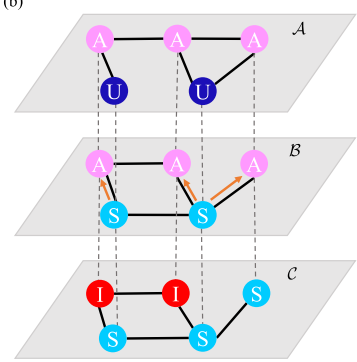

(c)
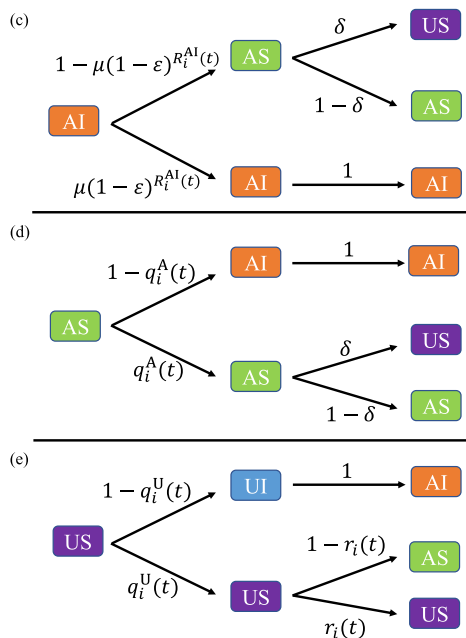

FIG. 1. Illustration of the information-resource-epidemic coevolution spreading model. (a) Mutual interactions among epidemic spreading, information diffusion, and resource allocation. (b) Proposed three-layer multiplex network, where the nodes are divided into unaware (U) and aware (A) states in the information layer $(\mathcal{A})$ and into susceptible $(\mathrm{S})$ and infected (I) states in the epidemic layer $(\mathcal{C})$. The orange arrows represent that susceptible individuals distribute resources to their aware neighbors in the resource layer $(\mathcal{B})$. The edges between nodes represent the online (or offline) social relationship in the information (or resource and epidemic) layer(s). The vertical dashed lines indicate the one-to-one correspondence among the nodes in the three layers. (c)-(e) The transition probability trees for the states AI, AS, and US, respectively, at time step $t$.

of resource allocation.) The resource amount possessed by individuals in turn affects the epidemic spreading process, where protection-focused resources contribute to reducing the infection probability of aware individuals, while cure-focused resources serve to enhance the recovery probability of infected individuals.

To model the complex interplay among information diffusion, resource distribution, and epidemic spreading, a multiplex network of three layers is appropriate, as shown in Fig. 1(b). Suppose there are $N$ individuals in the system, each belonging simultaneously to all three layers. Each network layer thus has $N$ nodes, and there is a one-to-one correspondence among the nodes in the three layers. For simplicity, we focus on the case where the networks in all three layers are unweighted and undirected. The processes of information diffusion, resource allocation, and epidemic spreading occur in the $\mathcal{A}, \mathcal{B}$, and $\mathcal{C}$ layers, respectively. In the information layer, the various edges represent the online or offline social relationships (e.g., friend relationships on Facebook). In the epidemic layer, the edges characterize the offline social relationships, e.g., with colleagues, family members, or neighbors. Resources are physically distributed via offline social relationships, rendering the identical topology of the resource and epidemic layers, as indicated in Fig. 1(b). Let $\mathbb{A}=\left[a_{i j}\right]$ be the adjacency matrix for the information layer: if there is a communication relation between individuals $i$ and $j$, we have $a_{i j}=1$ in the information layer; otherwise, $a_{i j}=0$. Similarly, let $\mathbb{B}=\left[b_{i j}\right]$ denote the matrix for the resource and epidemic layers: if individuals $i$ and $j$ have an offline relationship through which physical contacts occur, we have $b_{i j}=1$ in the resource and epidemic layers; otherwise, $b_{i j}=0$. The mean degree of the epidemic layer and the information layer are denoted by $\langle k\rangle$ and $\left\langle k_{1}\right\rangle$, respectively.

To quantitatively describe the dynamics in the information and epidemic layers, we adopt the standard unaware-awareunaware (UAU) [13] and susceptible-infected-susceptible (SIS) processes, respectively, where A (U) describes the situation where an individual is aware (unaware) of the epidemic, and I (S) specifies that an individual is infected (susceptible). After an epidemic starts, an individual can be informed of the virus spreading by an aware neighbor in the information layer. If an individual has already been infected, he or she becomes aware.

Mathematically, there are four state combinations: aware and infected (AI), aware and susceptible (AS), unaware and susceptible (US), and unaware and infected (UI). Since this paper focuses on how the interplay between information diffusion and resource distribution affects epidemic spreading, we follow the standard modeling approach by ignoring the latent time from the UI to the AI state and assuming that, once an individual is infected, he or she becomes aware of the disease immediately $[13,23]$. As a result, at any time during the process, an individual can be in one of the three different states: AI, AS, or US.

The coevolution of the dynamical processes in the three layers is described as follows. At the beginning of each time step $t$, infected individuals are unable to generate resources; only susceptible individuals, i.e., those in the AS or US state, are able to generate $r$ units of resources. For simplicity, we set $r=1$. Next, resource allocation occurs, followed by epidemic spreading and information diffusion.

In the resource layer, aware individuals seek resource support from their susceptible neighbors. As a result, any susceptible individual whose neighborhood contains at least one aware individual distributes one unit of resource equally to his or her aware neighbors and himself or herself. After resource allocation at time $t$, the resource amount possessed by individual $i$ is $R_{i}(t)$, which will affect the infection and the recovery probabilities.

In the epidemic layer, individual $i$ is infected by an infected neighbor $j$ with the probability $\beta$ if $i$ is in the US state at time $t$, but the probability becomes $\beta \varepsilon^{R_{i}(t)}$ if individual $i$ is in the AS state, where $0 \leqslant \varepsilon \leqslant 1$ is a parameter characterizing the efficacy of the resources. That is, if the individual is aware of the disease and has consequently secured a certain amount of resources to protect himself or herself, the infection probability will be reduced by the factor $\varepsilon^{R_{i}(t)}$. Once an individual in the US or AS state has been infected, the state of the individual will become $\mathrm{AI}$ at time $t+1$.

In the infected state, an individual $j$ will recover with the probability $1-\mu(1-\varepsilon)^{R_{j}(t)}$, where $\mu \in[0,1]$ is a parameter characterizing the dependence of recovery on medical resources. It is worth noting that this definition is different from the conventional one in which $\mu$ is directly the recovery probability. In our definition, the larger the value of $\mu$, the smaller the recovery probability. The strongest and weakest recovery probabilities occur for $\mu=0$ and 1 for fixed values of $\varepsilon$ and $R_{j}(t)$, respectively. 
If $j$ does not recover at $t$, he or she will remain in the AI state at $t+1$.

The physical meaning of the parameter $\varepsilon$ can be described as follows. If its value is close to zero, the main effect of the resources will be on the infection probability; it will be drastically reduced insofar as there is a certain amount of resources. However, a near zero $\varepsilon$ value means that the resources will have little effect on the recovery probability because it is approximately $(1-\mu)$ and independent of the resource amount. Resources of this type thus serve mainly the purpose of protection. On the contrary, if the value of $\varepsilon$ is close to one, the probability of recovery will increase with the resource amount, but the infection probability will hardly be affected. Resources of this type are cure focused.

In a word, aware individuals need to consume the received resources to protect themselves from being infected or to recover. For the individuals in the US state, most of their resources are allocated to their aware neighbors with the ongoing epidemic spreading. It is thus reasonable to assume that individuals consume all their resources at the current time step.

In the information layer, the spreading dynamics are of the standard SIS type; aware individuals inform their unaware neighbors with probability $\lambda$. An aware individual may forget or simply ignore the epidemic to switch into the $\mathrm{U}$ state and seek no resources, and this occurs with probability $\delta$. After information diffusion is completed at time $t$ to generate a new set of aware and unaware individuals, aware individuals will seek resource support from neighbors at the next time step.

\section{THEORETICAL ANALYSIS BASED ON MMC}

Our theoretical method consists of using the MMC theory [13] to analyze the coupled spreading dynamics in the threelayered network.

At each time step $t$, individual $i$ can be in one of three possible states: AI, AS, and US with probability $\rho_{i}^{\mathrm{AI}}(t), \rho_{i}^{\mathrm{AS}}(t)$, and $\rho_{i}^{\mathrm{US}}(t)$, respectively, where $\rho_{i}^{\mathrm{AI}}(t)+\rho_{i}^{\mathrm{AS}}(t)+\rho_{i}^{\mathrm{US}}(t)=$ 1. After resources have been allocated in the resource layer, the resource amount possessed by individual $i$ when he or she is in the $\mathrm{AI}$ (AS or US) state is $R_{i}^{\mathrm{AI}}(t)\left[R_{i}^{\mathrm{AS}}(t)\right.$ or $\left.R_{i}^{\mathrm{US}}(t)\right]$ at time $t$, which can be computed as

$$
\begin{gathered}
R_{i}^{\mathrm{AI}}(t)=\sum_{j} b_{i j} \rho_{j}^{\mathrm{S}}(t) \frac{1}{\sum_{l \neq i} b_{j l} \rho_{l}^{\mathrm{A}}(t)+2}, \\
R_{i}^{\mathrm{AS}}(t)=\sum_{j} b_{i j} \rho_{j}^{\mathrm{S}}(t) \frac{1}{\sum_{l \neq i} b_{j l} \rho_{l}^{\mathrm{A}}(t)+2} \\
\quad+\frac{1}{\sum_{j} b_{i j} \rho_{j}^{\mathrm{A}}(t)+1}, \\
R_{i}^{\mathrm{US}}(t)=\frac{1}{\sum_{j} b_{i j} \rho_{j}^{\mathrm{A}}(t)+1},
\end{gathered}
$$

where $\quad \rho_{j}^{\mathrm{S}}(t)=\rho_{j}^{\mathrm{AS}}(t)+\rho_{j}^{\mathrm{US}}(t), \quad$ and $\quad \rho_{l}^{\mathrm{A}}(t)=\rho_{l}^{\mathrm{AI}}(t)+$ $\rho_{l}^{\mathrm{AS}}(t)$.

For individual $i$ in the AI state, he or she can get resources from susceptible neighbors in the manner described by Eq. (1), where the denominator on the right side represents that the susceptible individual $j$ distributes resources to individual $i$, to himself or herself, and to other aware neighbors.
For individual $i$ in the AS state, the resource amount contains two parts: the amount received from susceptible neighbors and the remaining amount after distributing one unit of resources to every other aware individual. For individual $i$ in the US state, the resource amount is the amount left after distributing one unit of resources to every other aware neighbor. The mean resource amount of individuals in each state can be written as

$$
\left\langle R^{\mathrm{com}}\right\rangle(t)=\frac{\sum_{i} \rho_{i}^{\mathrm{com}}(t) R_{i}^{\mathrm{com}}(t)}{\sum_{i} \rho_{i}^{\mathrm{com}}(t)},
$$

where the superscript com represents the state combinations $\mathrm{AI}, \mathrm{AS}$, or US.

After the resource layer completes the allocation, both the amount of resources possessed by individual $i$ and the state he or she is in will affect the infection probability. The probability of individual $i$ not being informed by any aware neighbors in the information layer $r_{i}(t)$ and the probabilities of $i$ not being infected by any infected neighbors in the epidemic layer when he or she is aware and unaware $q_{i}^{\mathrm{A}}(t)$ and $q_{i}^{\mathrm{U}}(t)$, respectively, are

$$
\begin{gathered}
r_{i}(t)=\prod_{j}\left[1-a_{i j} \rho_{j}^{\mathrm{A}}(t) \lambda\right], \\
q_{i}^{\mathrm{A}}(t)=\prod_{j}\left[1-b_{i j} \rho_{j}^{\mathrm{AI}}(t) \beta \varepsilon^{R_{i}^{\mathrm{AS}}(t)}\right], \\
q_{i}^{\mathrm{U}}(t)=\prod_{j}\left[1-b_{i j} \rho_{j}^{\mathrm{AI}}(t) \beta\right],
\end{gathered}
$$

which constitute the base for constructing the transition probability trees shown in Figs. 1(c)-1(e), which illustrate the transition probabilities among the possible states after resource allocation, epidemic spreading, and information diffusion of each time step for individual $i$. This leads to the set of coupled MMC equations for the information-resourceepidemic dynamics as

$$
\begin{aligned}
\rho_{i}^{\mathrm{AI}}(t+1)= & \rho_{i}^{\mathrm{AI}}(t)\left[\mu(1-\varepsilon)^{R_{i}^{\mathrm{Al}}(t)}\right] \\
+ & \rho_{i}^{\mathrm{AS}}(t)\left[1-q_{i}^{\mathrm{A}}(t)\right]+\rho_{i}^{\mathrm{US}}(t)\left[1-q_{i}^{\mathrm{U}}(t)\right], \\
\rho_{i}^{\mathrm{AS}}(t+1)= & \rho_{i}^{\mathrm{AI}}(t)\left[1-\mu(1-\varepsilon)^{R_{i}^{\mathrm{AI}}(t)}\right](1-\delta) \\
& +\rho_{i}^{\mathrm{AS}}(t) q_{i}^{\mathrm{A}}(t)(1-\delta) \\
& +\rho_{i}^{\mathrm{US}}(t) q_{i}^{\mathrm{U}}(t)\left[1-r_{i}(t)\right], \\
\rho_{i}^{\mathrm{US}}(t+1)= & \rho_{i}^{\mathrm{AI}}(t)\left[1-\mu(1-\varepsilon)^{R_{i}^{\mathrm{AI}}(t)}\right] \delta \\
& +\rho_{i}^{\mathrm{AS}}(t) q_{i}^{\mathrm{A}}(t) \delta+\rho_{i}^{\mathrm{US}}(t) q_{i}^{\mathrm{U}}(t) r_{i}(t) .
\end{aligned}
$$

The fractions of aware and infected individuals at time $t, \rho^{\mathrm{A}}(t)$ and $\rho^{\mathrm{I}}(t)$, can be computed from

$$
\begin{aligned}
\rho^{\mathrm{A}}(t) & =\frac{\sum_{i}\left[\rho_{i}^{\mathrm{AS}}(t)+\rho_{i}^{\mathrm{AI}}(t)\right]}{N} \text { and } \\
\rho^{\mathrm{I}}(t) & =\frac{\sum_{i} \rho_{i}^{\mathrm{AI}}(t)}{N},
\end{aligned}
$$

where $N$ is the total number of individuals. 
When the system reaches a steady state, we have $\rho_{i}^{\mathrm{AI}}(t+$ 1) $=\rho_{i}^{\mathrm{AI}}(t)=\rho_{i}^{\mathrm{AI}}$, with a similar condition holding for $\rho_{i}^{\mathrm{AS}}(t+1), \rho_{i}^{\mathrm{US}}(t+1), R_{i}^{\mathrm{AI}}(t+1), R_{i}^{\mathrm{AS}}(t+1)$, and $R_{i}^{\mathrm{US}}(t+$ $1)$. Near the critical transmission probability $\beta_{c}$, the final probability that individual $i$ is in the infected state satisfies the relation $\rho_{i}^{\mathrm{AI}}=\theta_{i} \ll 1$. Neglecting high-order terms of $\theta_{j}$, we get

$$
\begin{aligned}
& q_{i}^{\mathrm{A}} \approx 1-\beta \varepsilon^{R_{i}^{\mathrm{AS}}} \sum_{j} b_{i j} \theta_{j}, \\
& q_{i}^{\mathrm{U}} \approx 1-\beta \sum_{j} b_{i j} \theta_{j} .
\end{aligned}
$$

Inserting these expressions into Eq. (8), we get

$\theta_{i}=\theta_{i}\left[\mu(1-\varepsilon)^{R_{i}^{\mathrm{AI}}}\right]+\rho_{i}^{\mathrm{AS}} \beta \varepsilon^{R_{i}^{\mathrm{AS}}} \sum_{j} b_{i j} \theta_{j}+\rho_{i}^{\mathrm{US}} \beta \sum_{j} b_{i j} \theta_{j}$.

With $\rho_{i}^{\mathrm{AS}}=\rho_{i}^{\mathrm{A}}-\rho_{i}^{\mathrm{AI}}$ and $\rho_{i}^{\mathrm{US}}=1-\rho_{i}^{\mathrm{A}}$, we rewrite Eq. (13), by neglecting the second-order term $-\theta_{i} \varepsilon^{R_{i}^{\mathrm{AS}}} \sum_{j} b_{i j} \theta_{j}$, as follows

$$
\sum_{j}\left\{\left[1-\left(1-\varepsilon^{R_{i}^{\mathrm{AS}}}\right) \rho_{i}^{A}\right] b_{i j}-\frac{1-\mu(1-\varepsilon)^{R_{i}^{\mathrm{AI}}}}{\beta} \delta_{i j}\right\} \theta_{j} \approx 0,
$$

where $\delta_{i j}$ is the element of the identity matrix.

In a multiplex network where the information and epidemic layers are different and possess a homogeneous structure (e.g., random or random-regular networks), the probability that individual $i$ is in the aware (or infected) state when the system has reached the steady state satisfies $\rho_{i}^{\mathrm{A}} \approx \rho^{\mathrm{A}}\left(\right.$ or $\rho_{i}^{\mathrm{I}} \approx \rho^{\mathrm{I}}$ ). The resource amount $R_{i}^{\mathrm{AS}}$ (or $R_{i}^{\mathrm{AI}}$ ) possessed by individual $i$ when he or she is in the AS (or AI) state can be expressed as

$$
\begin{gathered}
R_{i}^{\mathrm{AS}} \approx R^{\mathrm{AS}}=\langle k\rangle \rho^{\mathrm{S}} \frac{1}{(\langle k\rangle-1) \rho^{\mathrm{A}}+2}+\frac{1}{\langle k\rangle \rho^{\mathrm{A}}+1}, \\
R_{i}^{\mathrm{AI}} \approx R^{\mathrm{AI}}=\langle k\rangle \rho^{\mathrm{S}} \frac{1}{(\langle k\rangle-1) \rho^{\mathrm{A}}+2}
\end{gathered}
$$

where $\rho^{\mathrm{S}}=1-\rho^{\mathrm{I}}$, and $\langle k\rangle$ is the mean degree of the epidemic layer. Equation (14) can then be written as

$$
\sum_{j}\left\{\left[1-\left(1-\varepsilon^{R^{\mathrm{AS}}}\right) \rho^{\mathrm{A}}\right] b_{i j}-\frac{1-\mu(1-\varepsilon)^{R^{\mathrm{AI}}}}{\beta} \delta_{i j}\right\} \theta_{j} \approx 0 .
$$

Equation (17) thus becomes an eigenvalue problem for the matrix $\mathbb{H}$ given by

$$
\mathbb{H}=\left[1-\left(1-\varepsilon^{R^{\mathrm{AS}}}\right) \rho^{\mathrm{A}}\right] \mathbb{B},
$$

whose elements are

$$
h_{i j}=\left[1-\left(1-\varepsilon^{R^{\mathrm{AS}}}\right) \rho^{\mathrm{A}}\right] b_{i j} .
$$

The critical transmission probability $\beta_{c}$ is the minimum value $\beta$ for the onset of the epidemic and can be obtained as

$$
\begin{aligned}
\beta_{c} & \approx \frac{1-\mu(1-\varepsilon)^{R^{\mathrm{AI}}}}{\Lambda_{\max }(\mathbb{H})} \\
& =\frac{1-\mu(1-\varepsilon)^{R^{\mathrm{AI}}}}{\left[1-\left(1-\varepsilon^{R^{\mathrm{AS}}}\right) \rho^{\mathrm{A}}\right] \Lambda_{\max }(\mathbb{B})} \\
& \approx \frac{1-\mu(1-\varepsilon)^{R^{\mathrm{AI}}}}{\left[1-\left(1-\varepsilon^{R^{\mathrm{AS}}}\right) \rho^{\mathrm{A}}\right]\langle k\rangle},
\end{aligned}
$$

where $\Lambda_{\max }(\mathbb{H})$ and $\Lambda_{\max }(\mathbb{B})$ are the largest eigenvalues of the adjacency matrices $\mathbb{H}$ and $\mathbb{B}$, respectively. When the epidemic layer is a homogeneous network, the largest eigenvalue of its adjacency matrix is close to the mean degree of the network [33]: $\Lambda_{\max }(\mathbb{B}) \approx\langle k\rangle$. It is worth noting that the approximation of $\beta_{c}$ in Eq. (20) is based on the assumption that the information and epidemic layers are different, but they possess a homogeneous structure.

In Eq. (20), the dynamical process of information diffusion (i.e., $\rho^{\mathrm{A}}$ ) and resource allocation (i.e., $\varepsilon, R^{\mathrm{AS}}$, and $R^{\mathrm{AI}}$ ) will have an effect on the critical transmission probability.

We analyze the optimal type of resources to maximize the critical transmission probability. For $\mu=0, \beta_{c}$ decreases as $\varepsilon$ increases, so the optimal value of $\varepsilon$ is $\varepsilon_{\text {opt }}=0$. For $\mu>0$, we have

$$
\frac{\partial \beta_{c}}{\partial \varepsilon}=\frac{\mu R^{\mathrm{AI}}(1-\varepsilon)^{R^{\mathrm{AI}}-1}\left(1-\rho^{\mathrm{A}}+\rho^{\mathrm{A}} \varepsilon^{R^{\mathrm{AS}}}\right)-\left[1-\mu(1-\varepsilon)^{R^{\mathrm{AI}}}\right] \rho^{\mathrm{A}} R^{\mathrm{AS}} \varepsilon^{R^{\mathrm{AS}}-1}}{\left[1-\left(1-\varepsilon^{R^{\mathrm{AS}}}\right) \rho^{\mathrm{A}}\right]^{2}\langle k\rangle} .
$$

For a sufficiently large value of $\langle k\rangle$, both values of $R^{\mathrm{AI}}$ and $R^{\mathrm{AS}}$ are $>1$ (verified in Appendix A). Equation (21) stipulates that the value of $\partial \beta_{c} / \partial \varepsilon$ must be positive for $\varepsilon=0$ and negative for $\varepsilon=1$. An optimal value $\varepsilon_{\text {opt }}$ thus exists to maximize the critical transmission probability, which can be calculated from

$$
\left.\frac{\partial \beta_{c}}{\partial \varepsilon}\right|_{\varepsilon=\varepsilon_{\mathrm{opt}}}=0
$$

\section{RESULTS}

We simulated the information-resource-epidemic coevolution spreading model to unravel how information diffusion affects the epidemic dynamics under different resource support in real and synthetic multiplex networks. Our real multiplex network was taken from the combinatorial analysis of multiple networks [34], where the actual data consist of five kinds of online and offline social relationships: Facebook, leisure activities, work, coauthorship, and lunch meetings, among the computer science department employees at Aarhus. There are 61 nodes in total. We constructed the identical resource and epidemic layers by combining four out of the five types of social relationships: leisure activities, work, coauthorship, and lunch meetings, where the number of edges is 309 in each layer. The information layer is constructed based on all the online and offline social relations, where the number of edges is 353 . The parameters of the 
(a)

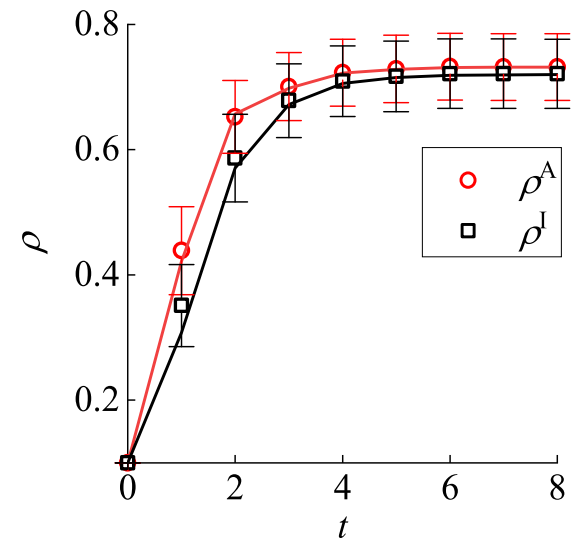

(b)

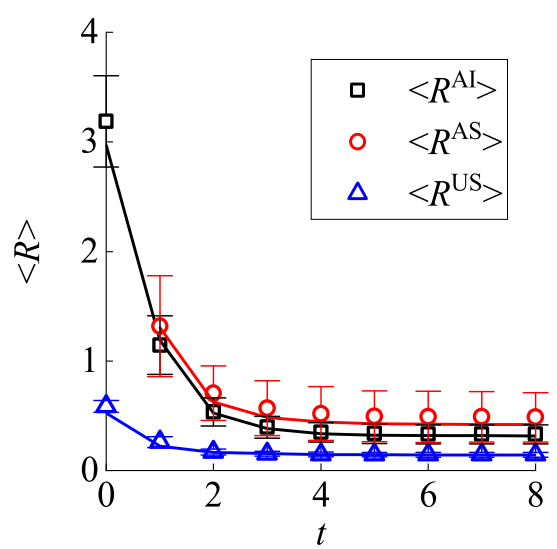

(c)

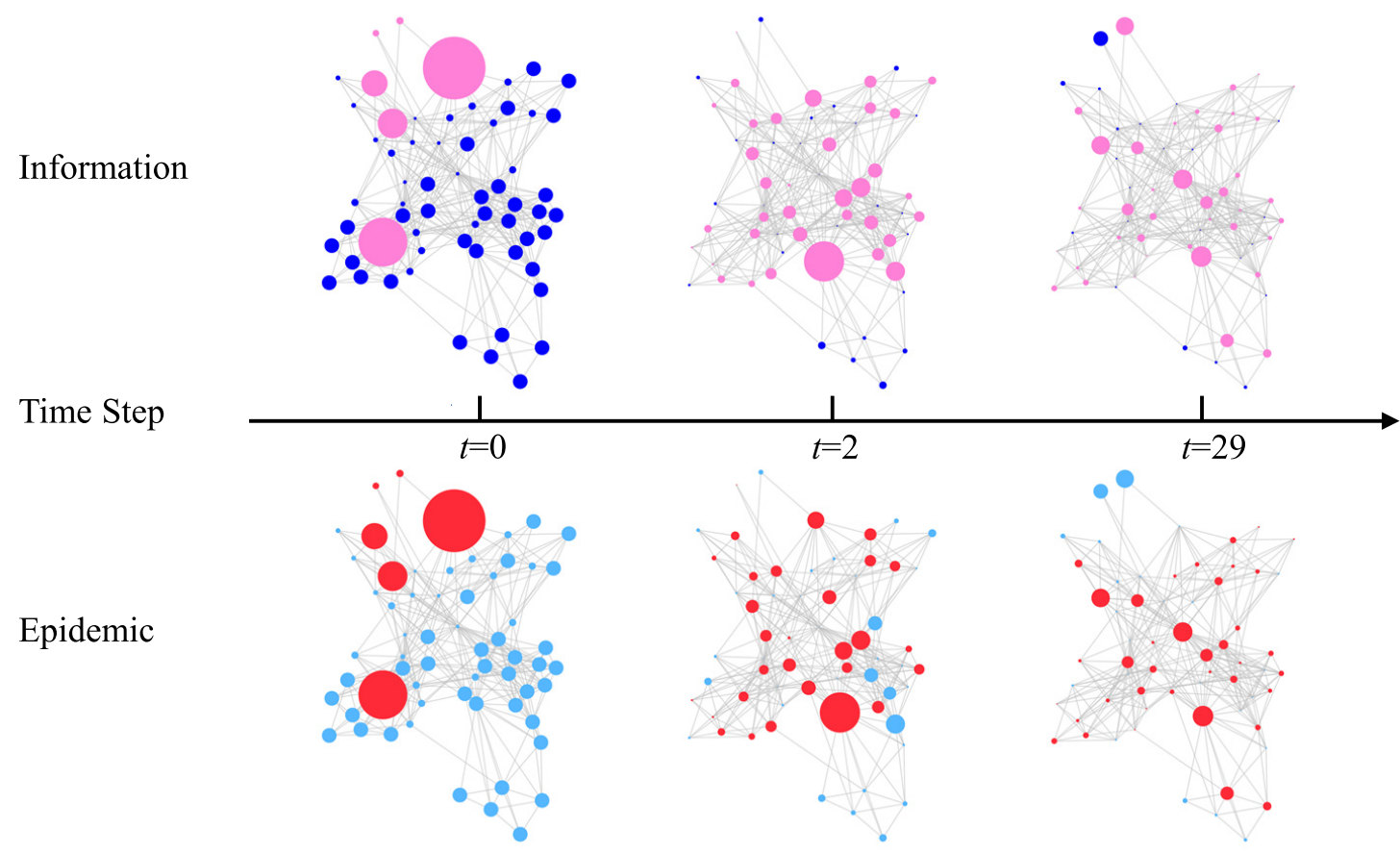

FIG. 2. Evolution of the resource amount of individuals in different states for the real three-layer multiplex network. (a) Evolution of the spreading sizes of the epidemic and information awareness, which increase rapidly initially and approach a steady value. (b) Time evolution of the average resource amount of individuals in different states. Each data point is the average result of 5000 realizations of a Monte Carlo simulation, and the solid traces are the predictions of the microscopic Markov chain (MMC) theory. The error bars indicate the standard deviations. (c) Visualization of the evolution of the dynamical state and the resource amount of each node at three time instants. In the information layer, the pink and blue colors represent the aware and unaware nodes, respectively. In the epidemic layer, the red and light blue colors denote the infected and susceptible individuals, respectively. The size of each node in the two layers represents the resource amount possessed by the node after resource allocation. The initial fraction $\rho_{0}$ of infected nodes is 0.1 . Other parameters are $N=61,\left\langle k_{1}\right\rangle=11.57$, $\langle k\rangle=10.13, \beta=0.4, \mu=0.8, \varepsilon=0.5, \lambda=0.2$, and $\delta=1.0$.

multiplex network and the degree distribution of each layer are illustrated in Appendix B.

It is useful to examine the time evolution of the resource amount in different states. Figure 2(a) shows a typical evolution pattern of the densities of infected and aware populations for a typical parameter setting, where both densities $\rho^{\mathrm{I}}$ and $\rho^{\mathrm{A}}$ increase with time rapidly and approach a respective constant value after a few time steps. The asymptotic constant density value of $\rho^{\mathrm{I}}$ is slightly smaller than that of $\rho^{\mathrm{A}}$, indicating a significant overlap between the aware and infected individuals. As the epidemic and aware sizes increase, the average resource amounts possessed by individuals in the three combined states (AI, AS, and US) decrease, as shown in Fig. 2(b). On average, individuals in the AS state possess the most resources, and those in the US state have the least. This behavior is consistent with the real situation where the aware and healthy individuals have more advantages by securing resource support than the individuals who are unaware or infected. The theoretical predictions agree with the simulation results.

At the initial stage $(t=0)$, there is only a small fraction of individuals in the AI state, and the others are in the US 
state. There are no individuals in the AS state when resource allocation starts at $t=0$, as it occurs at the beginning of each time step. As a result, the average resource amount of individuals in the AS state is calculated from $t=1$, as some susceptible individuals have been informed by this time.

To visualize the evolution of the resource distribution, we display the resource amount possessed by each node for three time instants from one realization of a Monte Carlo simulation, as shown in Fig. 2(c), which is represented by a circle at each node with its size indicating the resource amount. Initially $(t=0)$, there are a few aware and infected individuals who possess significantly more resources than other individuals in the information and epidemic layers. As the dynamics evolve, more individuals acquire resource support, and the average resource amount decreases.

We investigated the role of resource efficacy as characterized by the parameter $\varepsilon$ in epidemic spreading. Figures 3(a) and 3(b) show, for $\beta=0.15$ and 0.5 , respectively, $\rho^{\mathrm{I}}$ vs $\varepsilon$. In the regime of small values of $\varepsilon$ where resources are of the protection type, the asymptotic value of $\rho^{\mathrm{I}}$ depends strongly on the value of the recovery parameter $\mu$, where a small value of $\mu$ can lead to a small epidemic size. This is intuitively expected because of the relatively larger recovery probability in this case. Specifically, for a large value of $\mu$ (e.g., $\mu=0.8$ ), the epidemic size is large for a small value of $\varepsilon$, which indicates that, when the recovery probability is small, the protective type of resources is ineffective at suppressing the epidemic. As the value of $\varepsilon$ increases, the recovery probability is enhanced, and this effectively brings down the epidemic size, regardless of the value of the infection rate, as shown in Figs. 3(a) and 3(b). Comparing all six curves in Figs. 3(a) and $3(\mathrm{~b})$, we see that, if the value of $\mu$ is small so that the recovery probability is high, the epidemic size maintains at a small value in the full range of the $\varepsilon$ value, but if the value of $\mu$ is relatively large, insofar as the value of $\varepsilon$ is large enough to enhance the recovery probability, the epidemic size is also small. Note that, in Figs. 3(a) and 3(b), the data points are the result of direct network Monte Carlo simulations, but the various solid curves are not ones that connect the data points; they are actually the predictions of our MMC theory. The excellent agreement between numerics and theory exhibited in Figs. 3(a) and 3(b) attests to the validity and the predictive power of the MMC theory as applied to multiplex networks.

The curve of $\rho^{\mathrm{I}}$ vs $\varepsilon$ for the relatively small value of $\mu$ in Fig. 3(a) reveals a phenomenon: if the infection probability is low (e.g., $\beta=0.15$ ) and the recovery is mostly spontaneous without much medical help (e.g., $\mu=0.2$ ), there exists an optimal value of $\varepsilon$ for which the epidemic size is close to zero. To investigate this phenomenon in a more systematic way, we focus on a fundamental quantity underlying any epidemic spreading process: the critical value of the infection rate, denoted as $\beta_{c}$, above which there is an outbreak but below which the epidemic size approaches zero asymptotically. A larger value of $\beta_{c}$ signifies that the system requires a higher threshold infection probability to kick off an epidemic, deeming the system more resistant or resilient to any outbreak, which is desired. A viable way to assess the value of $\beta_{c}$ subject to resources is to calculate the epidemic size in the parameter plane $(\varepsilon, \beta)$ for any specific value of $\mu$. For computational feasibility, we take advantage of our MMC theory to make the (a)

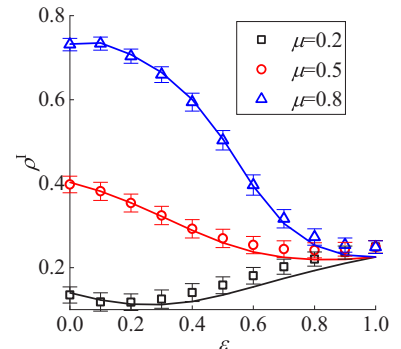

(c)

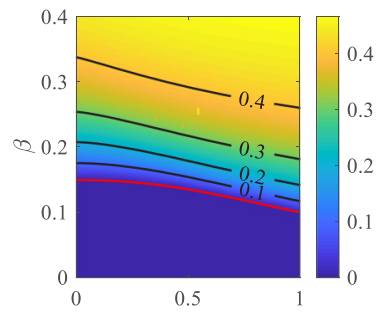

(e)

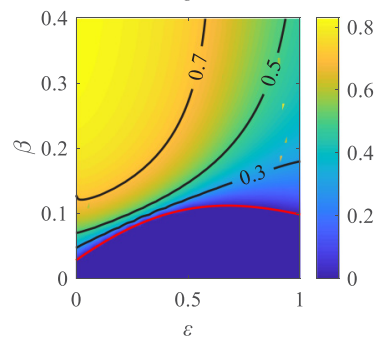

(b)

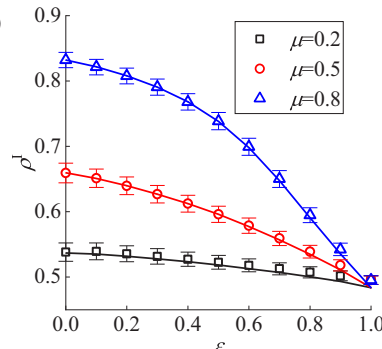

(d)

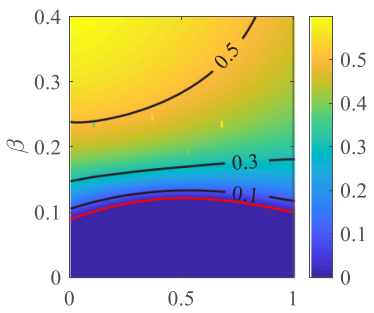

(f)

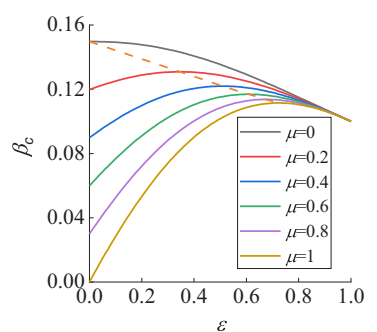

FIG. 3. Effects of resource efficacy on epidemic spreading. (a) and (b) The final epidemic size $\rho^{\mathrm{I}}$ vs $\varepsilon$ for three different values of $\mu$ for $\beta=0.15$ and 0.5 , respectively. Each data point is the result of averaging 5000 Monte Carlo realizations, and the curves are the theoretical predictions. The error bars represent standard deviations. (c)-(e) Color-coded values of $\rho^{\mathrm{I}}$ in the parameter plane $(\varepsilon, \beta)$ for $\mu=0,0.4$, and 0.8 , respectively, which are calculated from the microscopic Markov chain (MMC) theory. The black curves are contours of $\rho^{\mathrm{I}}$. The red curve gives the dependence of the critical infection rate $\beta_{c}$ on $\varepsilon$. (f) The curve of $\beta_{c}$ vs $\varepsilon$ for six different values of $\mu$. The position of the maximum value of $\beta_{c}$ on each curve is obtained from Eq. (22) in our MMC theory, and they are connected by the red dashed line. The initial infection density is $\rho_{0}=0.01$. The networks of information and epidemic layers have different random-regular (RR) topology, each of size $N=1000$ and average degree $\langle k\rangle=\left\langle k_{1}\right\rangle=10$. Other parameter values are $\lambda=0.2$ and $\delta=1.0$.

calculation. Figures 3(c)-3(e) show the color-coded values of the epidemic size in the $(\varepsilon, \beta)$ plane for three representative values of $\mu$, respectively, where the red curve in each case represents the dependence of $\beta_{c}$ on $\varepsilon$. For $\mu \neq 0$, an optimal type of resources emerges that maximizes the value of $\beta_{c}$, as exemplified in Figs. 3(d) and 3(e) and further demonstrated in Fig. 3(f), the plots of $\beta_{c}$ vs $\varepsilon$ for six values of $\mu$. Note that the optimal value of $\varepsilon$ increases as the value of $\mu$ is increased, reinforcing the observation that the recovery probability is key to suppressing the epidemic. Especially, for small values of $\mu$ that make the recovery rate high already, the optimal resource tends to be of the protection type. However, for large values of $\mu$ associated with which the spontaneous recovery rate without any resource is low, the optimal resource is of the cure type, which serves to enhance the overall recovery rate. 


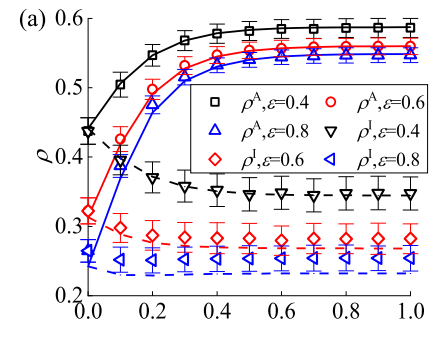

(c)

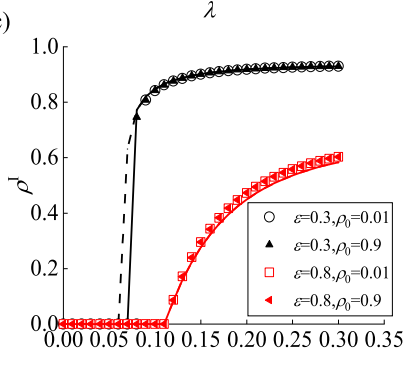

$\beta$

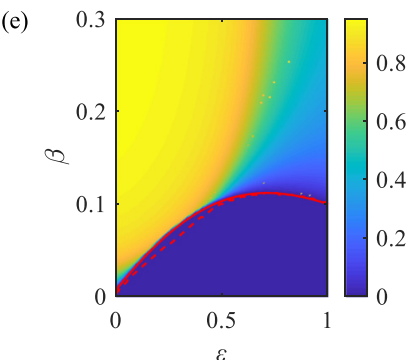

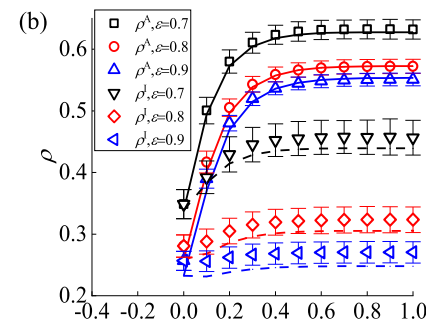

(d)

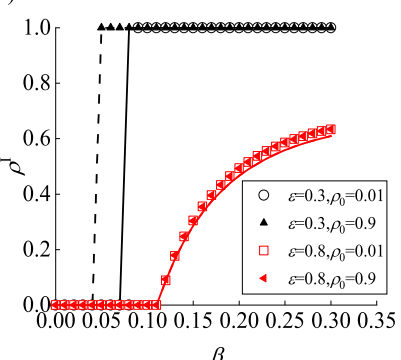

$\beta$

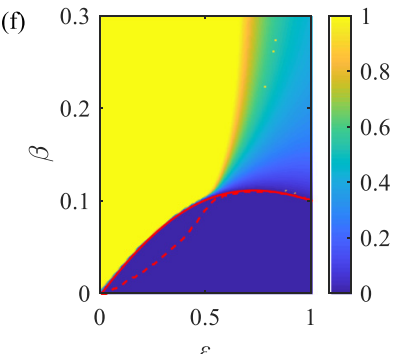

FIG. 4. Resource-induced anomalous epidemic behavior, phase transitions, and emergence of a hysteresis loop. (a) and (b) For $\mu=0.6$ and 1.0, respectively, information and epidemic spreading sizes ( $\rho^{\mathrm{A}}$ and $\rho^{\mathrm{I}}$, respectively) vs $\lambda$ for $\beta=0.15$ and $\rho_{0}=0.01$. As explained in the text, the behavior in (a) is expected, but that in (b) is anomalous. (c) and (d) The epidemic size vs $\beta$ for $\mu=0.95$ and 1.0, respectively. A second-order phase transition arises for $\varepsilon=0.8$, but the transition is of the first-order type for $\varepsilon=0.3$, associated with which is a hysteresis loop. (e) and (f) Color-coded value of $\rho^{\mathrm{I}}$ in the parameter plane $(\varepsilon, \beta)$ for $\mu=0.95$ and 1.0, respectively. Each data point is the result of averaging 5000 Monte Carlo realizations, and the solid and dashed curves are the theoretical predictions. The error bars indicate standard deviations. For (c)-(f), the value of $\lambda$ is fixed at 0.2 . The networks are of the random-regular (RR) type with $N=1000,\langle k\rangle=\left\langle k_{1}\right\rangle=10$, and $\delta=1.0$.

While the results in Figs. 3(c)-3(f) are obtained from a specific value of the initial infection density $\rho_{0}$, we have verified that the existence of an optimal type of resources to maximize the system resilience to epidemic spreading is not affected, even for larger values of $\rho_{0}$ (Appendix C).

How do the variations in the spreading probability $\lambda$ in the information layer affect the epidemic size and the phase transitions? It is common sense that the spread of the related information can raise the awareness of individuals and promote them to take protective measures, which helps to contain the epidemic. Simulation results testing this behavior are shown in Fig. 4(a) for $\mu=0.6$ and three values of $\varepsilon$. It can be seen that, as the value of $\lambda$ is increased, the final epidemic size $\rho^{\mathrm{I}}$ in the epidemic layer decreases continuously, as represented by the three dashed theoretical curves and the corresponding data points. Associated with the decrease in $\rho^{\mathrm{I}}$,

the final size $\rho^{\mathrm{A}}$ of aware individuals in the information layer increases continuously, as indicated by the three solid curves predicted by our theory and the corresponding simulation data points. However, as shown in Fig. 4(b), for a larger value of $\mu$, e.g., $\mu=1$.

As described in Appendix C, for $\varepsilon=0.5$, the corresponding resources are unable to contain the spreading. Consequently, a full outbreak will occur. In fact, the outbreak will persist for $\varepsilon<0.5$ because, as shown in Fig. 3(e), resource distribution characterized by a smaller $\varepsilon$ value will lead to a larger infection size for relatively large values of $\mu$. For $\varepsilon>0.5$ (e.g., $\varepsilon=0.7,0.8$, or 0.9 ), the resources are of the cure type. In this case, an anomalous behavior arises: as the spread in the information layer is intensified, more individuals in the epidemic layer are infected. The reason that an anomalous phenomenon occurs lies in the shortage of resources: as more individuals are aware of the epidemic, panic sets in, and they resort to an irrational acquisition behavior, thereby depleting the medical resources. The resource shortage even for frontline health workers leaves susceptible individuals more vulnerable to the disease, thereby increasing the epidemic size.

Our theory predicts another striking phenomenon, as verified by simulations, that an enhancement of the resource efficacy can lead to a characteristic change in the nature of the phase transition underlying the epidemic outbreak. In epidemiology, an outbreak as represented by a nonzero size of the final epidemic size $\rho^{\mathrm{I}}$ will not arise until the infection probability $\beta$ exceeds a critical value, i.e., $\beta_{c}$, giving rise to a phase transition. If $\rho^{\mathrm{I}}$ increases discontinuously and abruptly from zero to a positive value at $\beta_{c}$, the phase transition is of the first-order type. On the contrary, a continuous increase in the $\rho^{\mathrm{I}}$ value from zero as $\beta$ passes through $\beta_{c}$ leads to a second-order phase transition. Figures 4 (c) and 4(d) show, for $\mu=0.95$ and 1.0 , respectively, $\rho^{\mathrm{I}}$ vs $\beta$, each for two values of $\varepsilon$.

It can be seen that, in both panels, for $\varepsilon=0.8$, which corresponds to the situation where cure-focused resources are distributed, the phase transition is of the second-order type. However, for $\varepsilon=0.3$, where the resources allocated are of the protection type, the transition occurs at a smaller critical point, and it is of the first-order type.

In this case, the networked system becomes characteristically more prone to the epidemic, as even a weaker infection probability can cause the sudden emergence of a larger epidemic size. Associated with the first-order phase transition is a hysteresis loop, as indicated by the dashed curve of $\rho^{\mathrm{I}}$ vs $\beta$ in its descending direction.

The error bars of the simulation results in Figs. 4(c) and 4(d) are displayed in Appendix D. A more comprehensive picture of the epidemic outbreak can be obtained by examining systematically the theoretically predicted $\rho^{\mathrm{I}}$ value in the parameter plane $(\varepsilon, \beta)$, as shown by the color-coded value of $\rho^{\mathrm{I}}$ in Figs. 4(e) and 4(f) for $\mu=0.95$ and 1.0, respectively. In both cases, for large values of $\varepsilon$, in the vertical direction (i.e., as the value of $\beta$ is changed for a fixed value of $\varepsilon$ ), there is a gradual change in the color from dark blue to green or yellow and vice versa, signifying a second-order phase transition. However, for small values of $\varepsilon$, in the vertical direction, there is a sudden change in the color from dark blue to bright yellow 


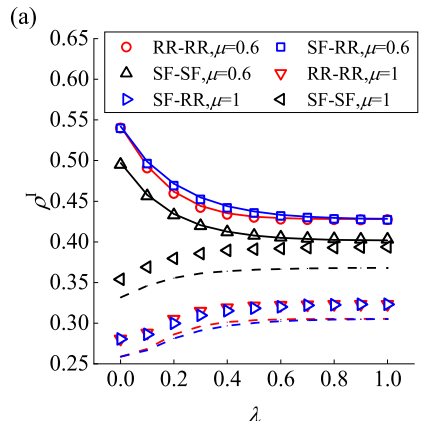

(c)

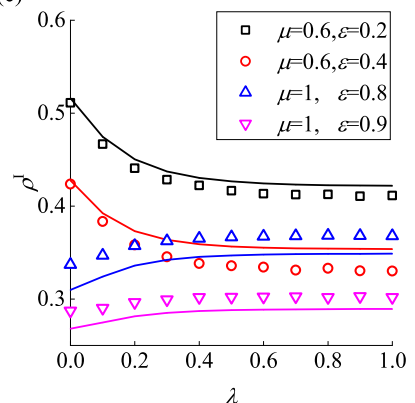

TABLE I. Details of the real three-layer multiplex network in our study.

\begin{tabular}{lcc}
\hline $\begin{array}{l}\text { Network } \\
\text { layer }\end{array}$ & $\begin{array}{c}\text { Resource or } \\
\text { epidemic layer }\end{array}$ & $\begin{array}{c}\text { Information } \\
\text { layer }\end{array}$ \\
\hline $\begin{array}{l}\text { Nodes } \\
\text { Edges }\end{array}$ & 61 & 61 \\
Relationship & 309 & 353 \\
& $\begin{array}{c}\text { Leisure activities, } \\
\text { Work, } \\
\text { Coauthorship, } \\
\text { Lunch gathering }\end{array}$ & $\begin{array}{c}\text { Facebook, } \\
\text { Leisure activities, } \\
\text { Work, } \\
\text { Coauthorship, } \\
\text { Lunch gathering }\end{array}$ \\
\hline \hline
\end{tabular}

However, a structural change in the epidemic layer affects the spreading more than a similar change in the information layer. When the epidemic layer is scale free, the infected hub nodes are more likely to recover for relatively small values of $\mu$ (e.g., $\mu=0.6$ ) and can get more resources to protect themselves, thereby reducing the risk of infection for low degree nodes to which the hub nodes are connected. As a result, the epidemic size in the SF-SF network is smaller than that in RR-RR or SF-RR networks. However, for $\mu=1$, it becomes harder for the infected hub nodes to recover, as resources have been allocated to others. Figure 5(b) shows $\rho^{\mathrm{I}}$ vs $\beta$ for $\lambda=0.2$ and $\mu=1$ for the SF-SF multiplex network, revealing a first-order phase transition and a hysteresis loop. Qualitatively similar phenomena occur for the real-world network, as shown in Figs. 5(c) and 5(d).

\section{DISCUSSION}

Epidemic spreading in the real world is not an isolated process in the physical contact space, but it is always accompanied by the diffusion of the pertinent information in cyberspace. The dissemination of information motivates individuals to seek protection or cure resources, thereby triggering resource allocation among individuals. The resource amount possessed by an individual will affect his or her infection and recovering probabilities, modulating the epidemic spreading dynamics. The interplay and coevolution among information diffusion, resource allocation, and epidemic spreading are extraordinarily complex, an understanding of which is critical
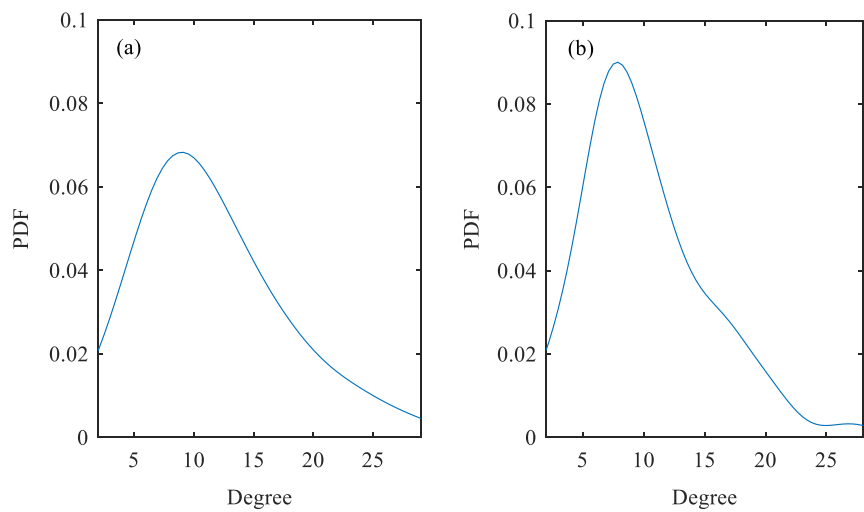

FIG. 6. Degree distribution of (a) the information layer and (b) the epidemic layer. 

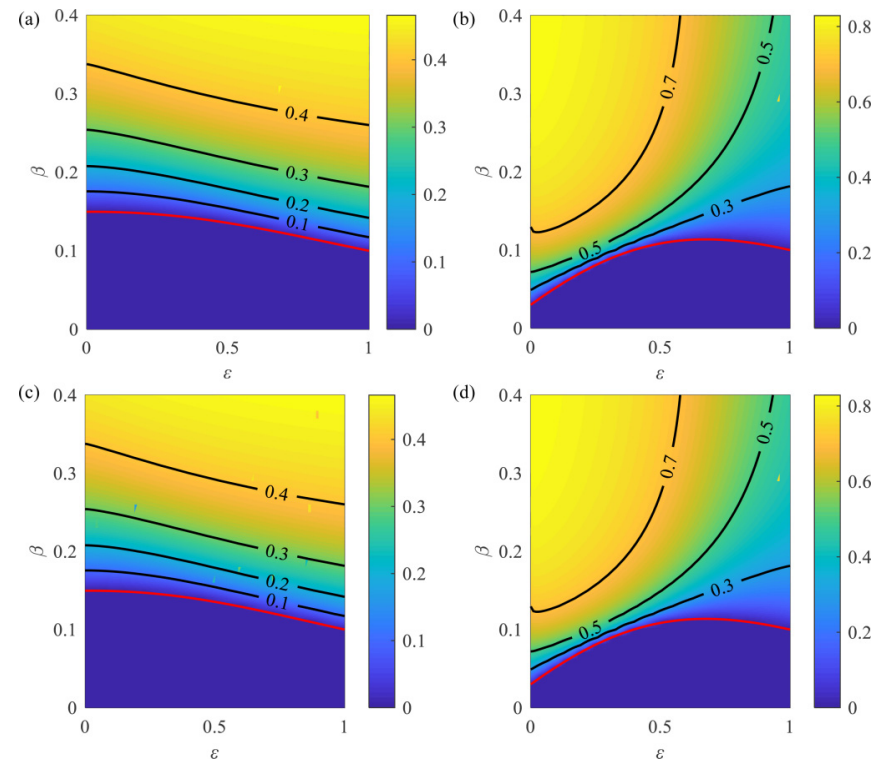

FIG. 7. Effect of resource efficacy parameter $\varepsilon$ on epidemic spreading. The color-coded values $\rho^{\mathrm{I}}$ predicted by the microscopic Markov chain (MMC) theory in the parameter plane $(\varepsilon, \beta)$ for (a) $\mu=0$ and $\rho_{0}=0.05$, (b) $\mu=0.8$ and $\rho_{0}=0.05$, (c) $\mu=0$ and $\rho_{0}=0.1$, and (d) $\mu=0.8$ and $\rho_{0}=0.1$. The black curves are the contours of $\rho^{\mathrm{I}}$. The red curves indicate the change of $\beta_{c}$. The information and epidemic layers are different random-regular (RR) networks. Other parameter values are $N=1000,\langle k\rangle=\left\langle k_{1}\right\rangle=10$, $\lambda=0.2$, and $\delta=1.0$.

to predicting the spreading trend of the epidemic and making effective strategies for containing the epidemic.

We have constructed a three-layer network model to describe the information-resource-epidemic coevolution spread-
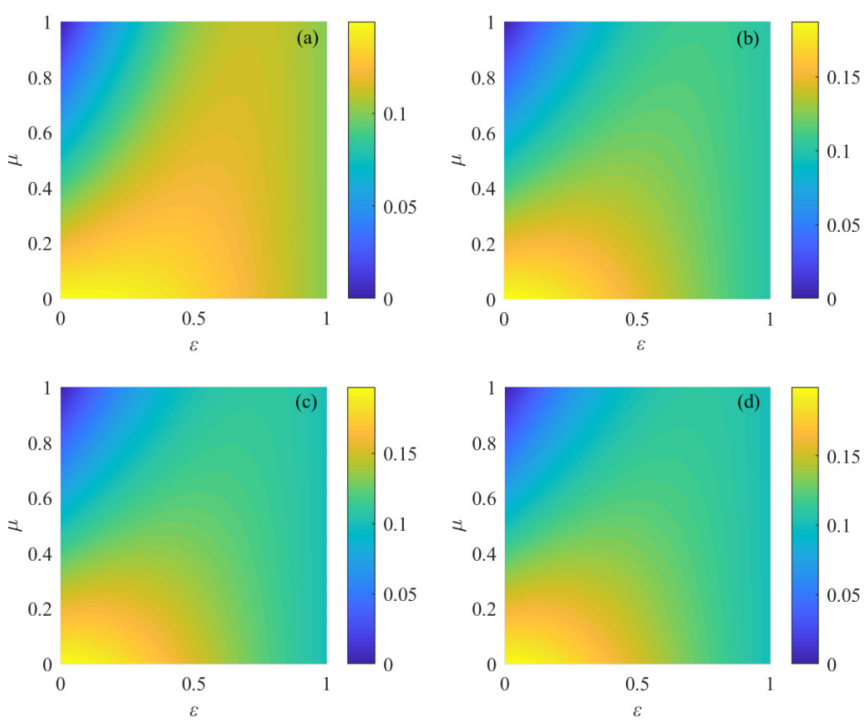

FIG. 8. Dependence of the critical phase transition point on resource type and recovery parameter. Shown is the dependence of $\beta_{c}$ on $\varepsilon$ and $\mu$ for (a) $\lambda=0.2$, (b) $\lambda=0.4$, (c) $\lambda=0.6$, and (d) $\lambda=0.8$. The information and epidemic layers are different random-regular (RR) networks. Other parameter values are $N=1000,\langle k\rangle=\left\langle k_{1}\right\rangle=$ 10 , and $\delta=1.0$.
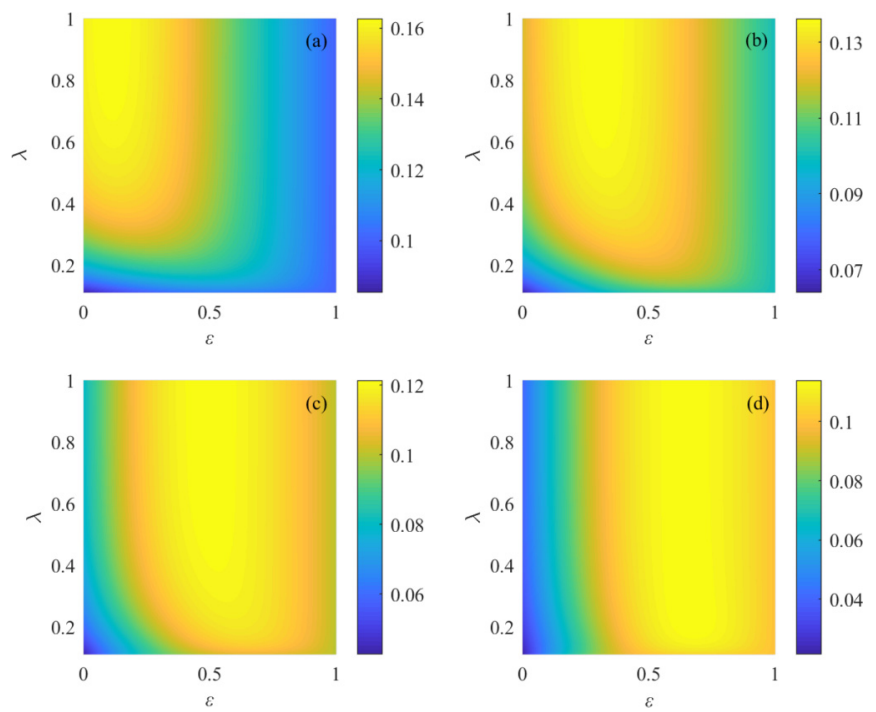

FIG. 9. Dependence of the critical phase transition point on resource type and information spreading rate. Shown is the dependence of $\beta_{c}$ on $\varepsilon$ and $\lambda$ for (a) $\mu=0.2$, (b) $\mu=0.4$, (c) $\mu=0.6$, and (d) $\mu=0.8$. The information and epidemic layers are different random-regular (RR) networks. Other parameter values are $N=$ $1000,\langle k\rangle=\left\langle k_{1}\right\rangle=10$, and $\delta=1.0$.

ing process. The basic model assumptions are two. First, information diffusion triggers individuals to seek resource support from their neighbors. Second, the amount and nature of resources possessed by an individual will affect his or her infection and recovering probabilities. We have carried out simulations using synthetic and real multiplex networks and developed a theoretical analysis. As a result of resource allocation triggered by information diffusion, aware and susceptible individuals tend to secure more resources than individuals in other epidemic states. Depending on the recovery probability, the optimal type of resources to suppress epidemic spreading can be different. If this probability is relatively large, the protection-focused type of resources is effective at reducing the epidemic size, and the phase transition underlying the outbreak is of the second-order type. However, if the recovery probability is small, cure-focused resources are more effective. In this case, the anomalous phenomenon that information diffusion worsens epidemic spreading can arise, due to the shortage of resources needed for infected individuals to recover. In addition, the phase transition becomes first order with a hysteresis loop when the allocated resources are protection focused. Signatures of the anomalous effect of information diffusion might have been present in the beginning phase of the ongoing COVID-19 pandemic, and our modeling framework and theory have provided a preliminary understanding in an idealized but quantitative setting.

\section{ACKNOWLEDGMENTS}

This paper was supported by the National Natural Science Foundation of China (Grant Nos. 61932005 and 61903266), the Fundamental Research Funds for the Central Universities with Contract No. 2019XD-A10, China Postdoctoral Science Foundation (Grant Nos. 2018M631073 and 2020M670233), 


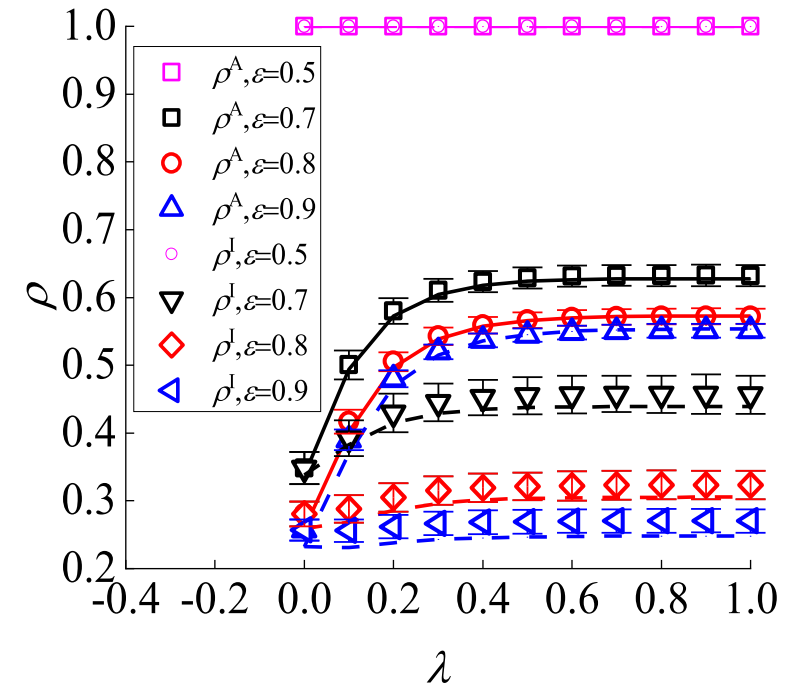

FIG. 10. Supplementary information for Fig. 4(b). Shown are information and epidemic spreading sizes $\left(\rho^{\mathrm{A}}\right.$ and $\rho^{\mathrm{I}}$, respectively) vs $\lambda$ for $\mu=1, \beta=0.15, \rho_{0}=0.01$, and $\delta=1.0$. The networks are of the random-regular (RR) type with $N=1000$ nodes and average degree $\langle k\rangle=\left\langle k_{1}\right\rangle=10$.

the Science and Technology Department of Sichuan Province (Nos. 2020YJ0048 and 2020YFS0007), and the Scientific Research Level Improvement Project to Promote the Colleges Connotation Development of Beijing Information Science \& Technology University in 2020 (Grant No. 2020KYNH212). YCL would like to acknowledge support from the Vannevar Bush Faculty Fellowship program sponsored by the Basic Research Office of the Assistant Secretary of Defense for Research and Engineering and funded by the Office of Naval Research through Grant No. N00014-16-1-2828.

\section{APPENDIX A: THEORETICAL ANALYSIS OF THE FINAL RESOURCE AMOUNT}

This Appendix presents the theoretical analysis of the final resource amount.

Near the critical transmission probability of the epidemic, we have $\rho^{\mathrm{S}}=1-\rho^{\mathrm{I}} \approx 1$, and the dynamics in the information layer are decoupled from those in the other layers. For information diffusion in a homogeneous network, the change in the fraction of aware individuals can be represented as

$$
\rho^{\mathrm{A}}(t+1)=\rho^{\mathrm{A}}(t)(1-\delta)+\left[1-\rho^{\mathrm{A}}(t)\right]\left[1-r_{1}(t)\right],
$$

where $r_{1}(t)$ is the probability that each node is not being informed by any aware neighbors at time step $t$, which is

$$
r_{1}(t) \approx\left[1-\rho^{\mathrm{A}}(t) \lambda\right]^{\left\langle k_{1}\right\rangle},
$$

where $\left\langle k_{1}\right\rangle$ is the mean degree of the information layer. For simplicity, we set $\delta=1$ [35]. When the diffusion process is stabilized, we have $\rho^{\mathrm{A}}(t+1)=\rho^{\mathrm{A}}(t)$ and get

$$
\rho^{\mathrm{A}}=1-\frac{1}{2-r_{1}} \leqslant 0.5 \text {. }
$$

When the network topology of the epidemic layer is homogeneous with a mean degree $>3$, the resource amount possessed
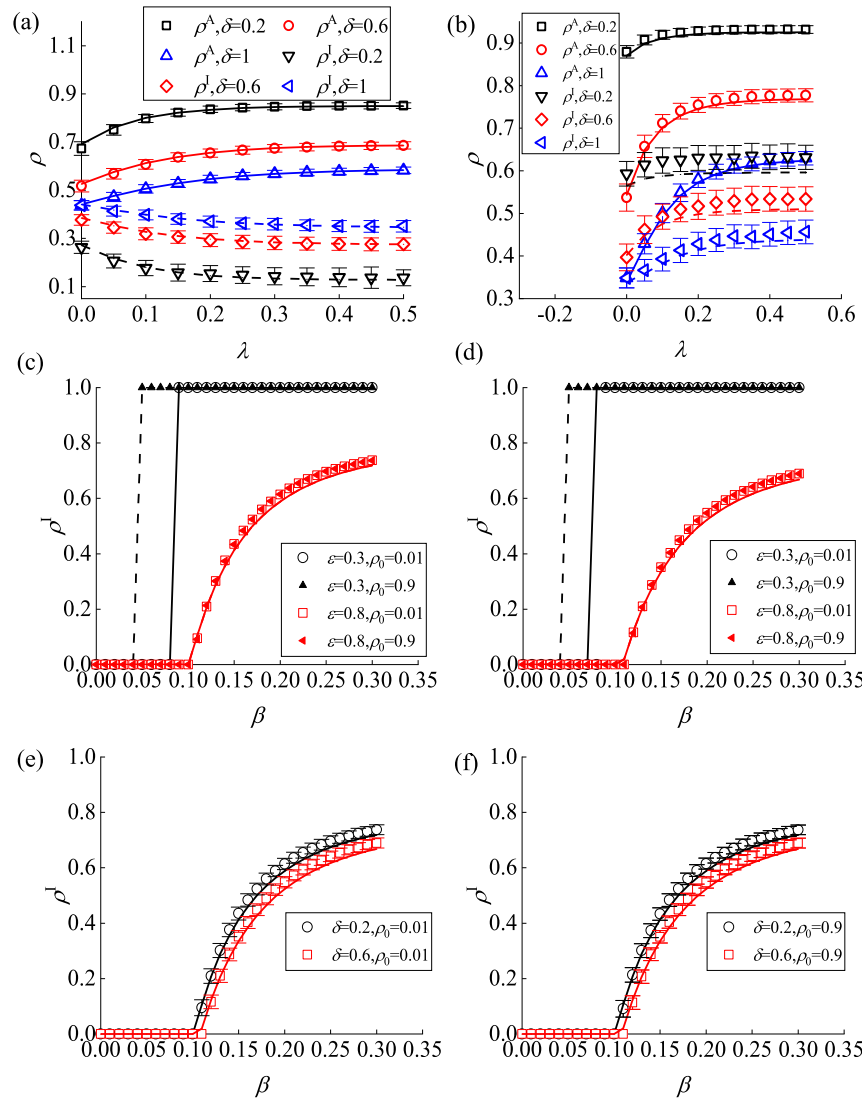

FIG. 11. Effects of the probability $\delta$ that an aware individual forgets or ignores the information on the coevolution spreading dynamics. (a) Information and epidemic spreading sizes $\left(\rho^{\mathrm{A}}\right.$ and $\rho^{\mathrm{I}}$, respectively) vs $\lambda$ for three different values of $\delta$. Other parameter values are $\mu=0.6, \varepsilon=0.4, \beta=0.15$, and $\rho_{0}=0.01$. (b) Same as (a) but for $\mu=1.0$ and $\varepsilon=0.7$. (c) and (d) For $\delta=0.2$ and 0.6 , respectively, the epidemic size vs $\beta$ for $\mu=1$ and $\lambda=0.2$. Each data point is the result of averaging 5000 Monte Carlo realizations, and the solid and dashed curves are the theoretical predictions. (e) and (f) The corresponding results with error bars for $\varepsilon=0.8$. For $\delta=0.2$ and 0.6 , the error bars for $\varepsilon=0.3, \rho_{0}=0.01$, and $\rho_{0}=0.9$ are negligible. The networks are of the random-regular (RR) type with $N=1000$ nodes and average degree $\langle k\rangle=\left\langle k_{1}\right\rangle=10$.

by each node in the AI state is

$$
R^{\mathrm{AI}} \approx \frac{\langle k\rangle}{(\langle k\rangle-1) \rho^{\mathrm{A}}+2} \geqslant \frac{\langle k\rangle}{(\langle k\rangle-1) \times 0.5+2}>1 .
$$

In this case, $R^{\mathrm{AS}}$ is also $>1$.

\section{APPENDIX B: REAL MULTIPLEX NETWORK DESCRIPTION}

This Appendix presents a detailed description of the real multiplex network used in our study.

The real data are about the relationship among the employees at the Department of Computer Science at Aarhus University. The data were collected from 61 employees, which included professors, postdoctoral researchers, Ph.D. students, and administration staff. Five different types of relations were studied [34], which are the current working relationship, re- 


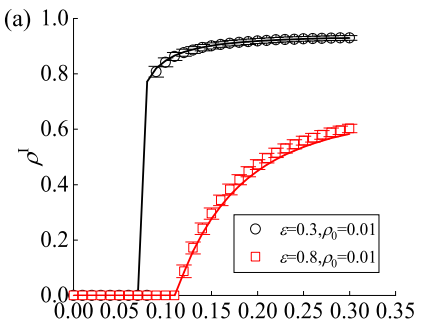

(c)

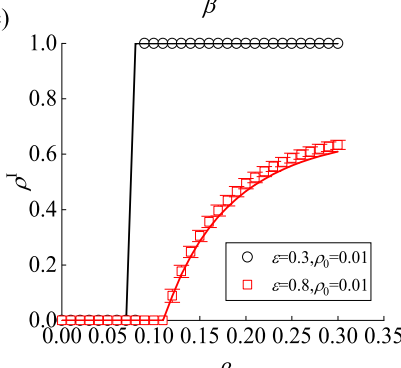

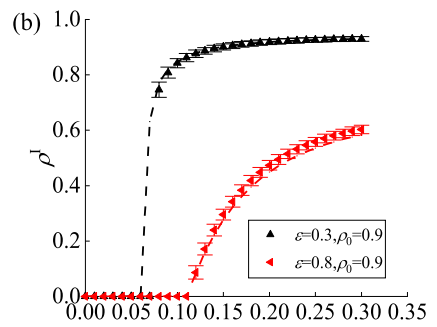

(d)

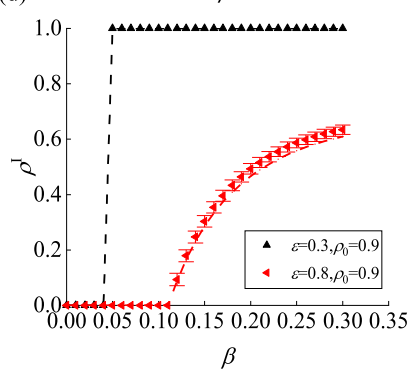

FIG. 12. The results in Figs. 4(c) and 4(d) but with error bars. (a) and (b) The epidemic size vs $\beta$ for $\mu=0.95$ and two different values of $\rho_{0}$, respectively. (c) and (d) The same as in (a) and (b) but for $\mu=1$, where the error bars are negligible for $\varepsilon=0.3$. In all panels, a data point is the result of averaging 5000 Monte Carlo realizations, and the solid and dashed curves are the theoretical predictions. The networks are of the random-regular (RR) type with $N=1000$ nodes and average degree $\langle k\rangle=\left\langle k_{1}\right\rangle=10$.

peated leisure activities, regular lunch meetings, coauthorship of publications, and their friendship relation on Facebook. Using these data, we constructed a three-layer network to study the information-resource-epidemic coevolution spreading dynamics. The 61 employees appear simultaneously in each layer. The edges among these individuals in the information layer are the combination of the five different types of relations, and the maximum (minimum) degree is 29 (2). The resource and epidemic layers have the same network topology, where the edges represent the social relationships of the current working relationship, repeated leisure activities, regular lunch meetings, and coauthorship of publications. The maximum (minimum) degree is 27 (2) in each layer. The details of the real multiplex network are in Table I. The distribution of degree of the information and epidemic layers is shown in Fig. 6.

\section{APPENDIX C: SPREADING PARAMETERS AND RESOURCE TYPE}

In the main text, the effect of the resource efficacy parameter $\varepsilon$ on final epidemic size has been analyzed. Here, we provide additional results from different initial values of the seed infection size $\rho_{0}$. For $\rho_{0}=0.05$, the final epidemic size increases with $\varepsilon$ for $\mu=0$, as shown in Fig. 7(a). However, for $\mu=0.8$, a smaller value of $\varepsilon$ will make the epidemic size larger, as shown in Fig. 7(b). These results are consistent with those in the main text. Similar results have been obtained for $\rho_{0}=0.1$, as shown in Figs. 7(c) and 7(d).

To unveil how resources affect the critical transmission probability $\beta_{c}$ in a more systematic manner, we calculate the values of $\beta_{c}$ in the parameter plane $(\varepsilon, \mu)$ for a different value
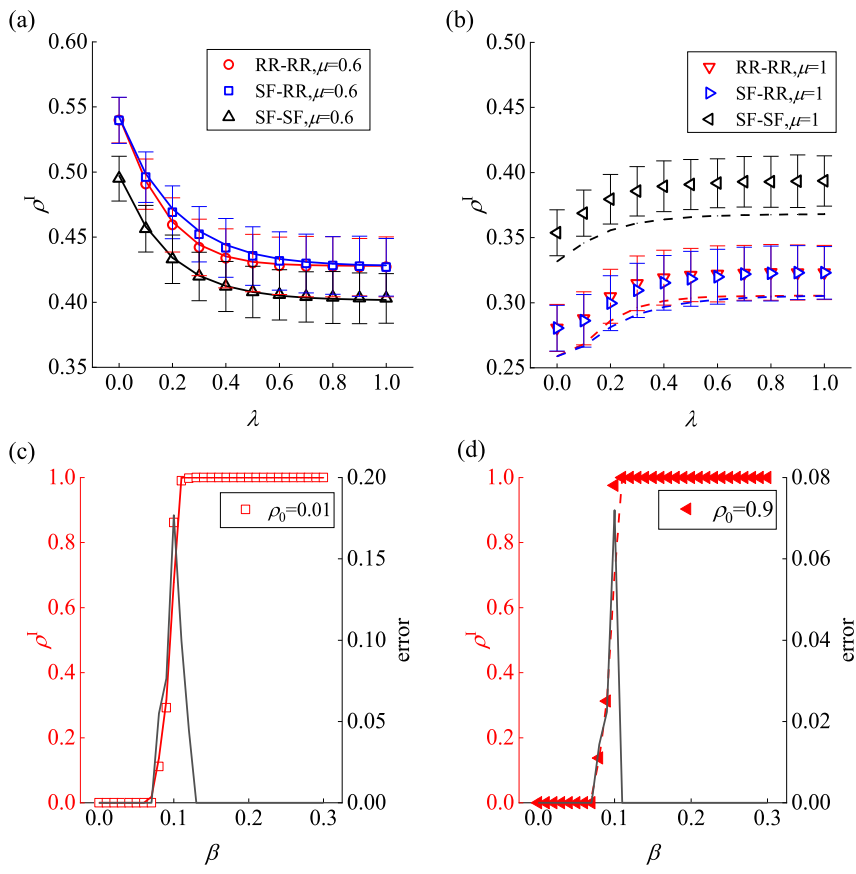

FIG. 13. The results in Figs. 5(a) and 5(b) but with error bars. (a) Epidemic size $\rho^{\mathrm{I}}$ vs $\lambda$ for $\varepsilon=0.2, \beta=0.15$, and $\rho_{0}=0.01$ in three types of combinations of information-epidemic layers: RR-RR, SF-RR, and SF-SF, where RR and SF stand for random-regular and scale-free, respectively. (b) Same as (a) but for $\varepsilon=0.8$. In (a) and (b), the curves (solid or dashed) are the predictions of the microscopic Markov chain (MMC) theory. The error bars indicate the standard deviations. (c) and (d) Epidemic size $\rho^{\mathrm{I}}$ vs $\beta$ for $\varepsilon=0.5, \lambda=0.2$, and $\mu=1$ in the SF-SF type of multiplex network for $\rho_{0}=0.01$ and 0.9 , respectively, where the red curves (solid or dashed) are the predictions of the MMC theory, and the black curves represent the errors. In all panels, a data point is the result of averaging 5000 Monte Carlo realizations. Other parameters are network size $N=1000$, the algebraic exponent of degree distribution 3 , average degrees $\langle k\rangle=\left\langle k_{1}\right\rangle=10$, and $\delta=1.0$.

of the information spreading rate, based on the theoretical analysis in the main text. We find that the optimal value $\varepsilon_{\text {opt }}$ that maximizes $\beta_{c}$ increases with $\mu$ for different values of $\lambda$, as shown in Fig. 8. The results indicate that protection-focused resources are effective for containing epidemic spreading for relatively small values of $\mu$, but cure-focused resources are more effective for large values of $\mu$.

In addition, we studied the dependence of $\beta_{c}$ on $\varepsilon$ and $\lambda$ for different values of $\mu$, as shown in Fig. 9, with the result that the optimal resource type depends on the value of $\mu$, which is consistent with the result in the main text. Moreover, as the value of $\lambda$ is increased, the threshold for epidemic spreading is high for relatively small values of $\mu$, e.g., $\mu=0.2$ or 0.4 , as shown in Figs. 9(a) and 9(b), respectively. However, increasing the value of $\lambda$ has no significant effect on the value of $\beta_{c}$ for relatively large values of $\mu$, e.g., $\mu=0.6$ or 0.8 , as shown in Figs. 9(c) and 9(d), respectively.

Based on Fig. 4(b), we obtained results for the case of $\varepsilon=0.5$ in Fig. 10 with the finding that this type of resource distribution is unable to contain epidemic spreading. In fact, a full outbreak will occur. 


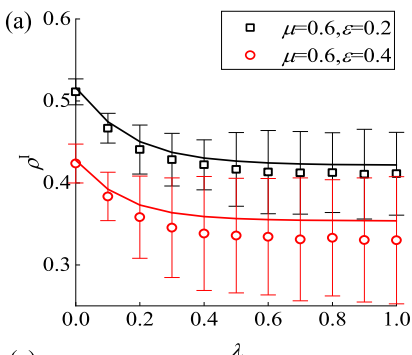

(c)

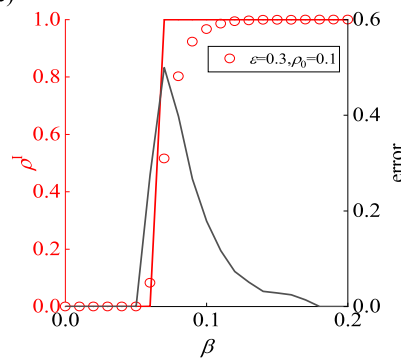

(e)

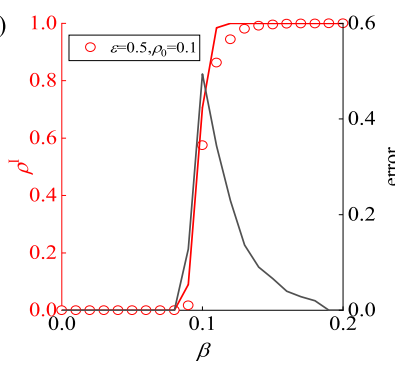

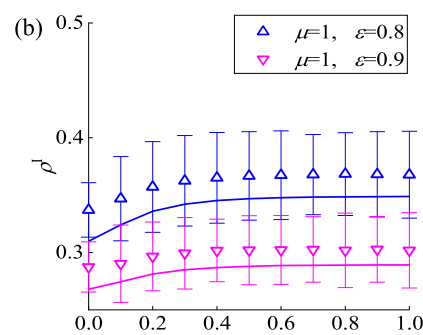

(d)
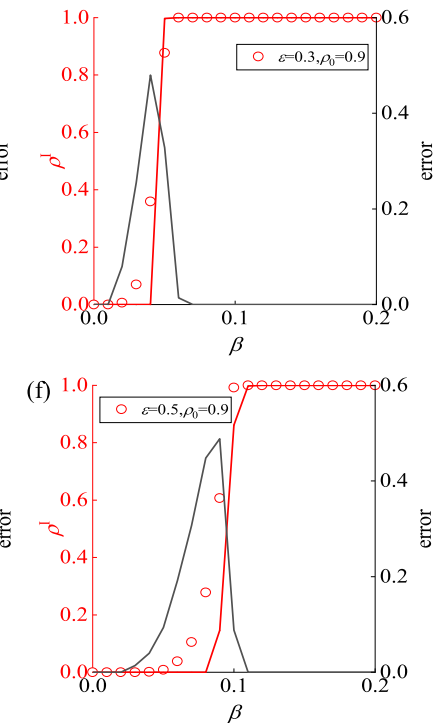

FIG. 14. Results of Figs. 5(c) and 5(d) but with error bars. (a) and (b) Epidemic size $\rho^{\mathrm{I}}$ vs $\lambda$ in the real-world multiplex network in Fig. 2 for $\beta=0.15$ and $\rho_{0}=0.1$. (c)-(f) Epidemic size $\rho^{\mathrm{I}}$ vs $\beta$ for the network in Fig. 2 for $\lambda=0.2$ and $\mu=1$, where the red curves are the predictions of the microscopic Markov chain (MMC) theory, and the black curves represent the errors. In all panels, a data point is the result of averaging 5000 Monte Carlo realizations, and $\delta=1.0$.

[1] A. R. McLean, R. M. May, J. Pattison, and R. A. Weiss, SARS: A Case Study in Emerging Infections (Oxford University Press, New York, 2005).

[2] C. Fraser et al., Pandemic potential of a strain of influenza A (H1N1): early findings, Science 324, 1557 (2009).

[3] M. Chinazzi, J. T. Davis, M. Ajelli, C. Gioannini, M. Litvinova, S. Merler, A. P. Y. Piontti, K. Mu, L. Rossi, K. Sun, C. Viboud, X. Xiong, H. Yu, M. E. Halloran, I. M. Longini, Jr., and A. Vespignani, The effect of travel restrictions on the spread of the 2019 novel coronavirus (COVID-19) outbreak, Science $\mathbf{3 6 8}$, 395 (2020)

[4] S. M. Kissler, C. Tedijanto, E. Goldstein, Y. H. Grad, and M. Lipsitch, Projecting the transmission dynamics of SARS$\mathrm{CoV}-2$ through the postpandemic period, Science 368, 860 (2020).

[5] J. J. V. Bavel et al., Using social and behavioural science to support COVID-19 pandemic response, Nat. Human Behav. 4, 460 (2020).

[6] WHO Director-General's Opening Remarks at the Media Briefing on 2019 Novel Coronavirus-7 February 2020 (World Health Organization, 2020).

In the main text, how information diffusion affects the epidemic size for different values of $\mu$ was studied for fixed $\delta=1.0$, with the result that information diffusion can either contain or promote epidemic spreading. Here, we study if choosing different values of $\delta$ would affect this result. Figure 11(a) shows, for different values of $\delta$, the final epidemic size $\rho$ vs $\lambda$, where the values of parameters $\mu$ and $\varepsilon$ are set to be the same as those in Fig. 4. We see that information diffusion can suppress epidemic spreading. As the value of $\delta$ decreases, aware individuals can stay in this state longer, raising the awareness of seeking protection and reducing the final epidemic spreading size. Figure 11(b) shows, for $\mu=1$ and $\varepsilon=0.7$ [the same parameter values as those in Fig. 4(b)], information diffusion can aggravate epidemic spreading. This is because the irrational acquisition behavior of aware individuals in this case can deplete the medical resources. The epidemic size is larger for smaller values of $\delta$. The phase transition behavior is also robust, as shown in Fig. 11(c) for $\delta=0.2$ and in Fig. 11(d) for $\delta=0.6$. For cure-focused $(\varepsilon=0.8)$ and protection-focused $(\varepsilon=0.3)$ resources, the phase transitions are of the second-order and first-order nature, respectively. The curves associated with the second-order transition with error bars included are shown in Figs. 11(e) and 11(f) for two different values of $\rho_{0}$. The results in Figs. 11(a)-11(f) indicate that the findings presented in the main text are robust against the choice of the value of $\delta$.

\section{APPENDIX D: ERROR BARS}

The results of Figs. 4(c) and 4(d) with error bars are displayed in Fig. 12. The results of Figs. 5(a) and 5(b), and 5(c) and 5(d), with errors are displayed in Figs. 13 and 14, respectively. The error bars are obtained from 5000 Monte Carlo realizations. For $\varepsilon=0.3$, the error bars for the SF-SF type of multiplex network are negligible.
[7] A. Vespignani, H. Tian, C. Dye, J. O. Lloyd-Smith, R. M. Eggo, M. Shrestha, S. V. Scarpino, B. Gutierrez, M. U. G. Kraemer, J. Wu, K. Leung, and G. M. Leung, Modelling COVID-19, Nat. Rev. Phys. 2, 279 (2020).

[8] C. Perrings, C. Castillochavez, G. Chowell, P. Daszak, E. P. Fenichel, D. Finnoff, R. D. Horan, A. M. Kilpatrick, A. P. Kinzig, N. V. Kuminoff, S. Levin, B. Morin, K. F. Smith, and M. Springborn, Merging economics and epidemiology to improve the prediction and management of infectious disease, Ecohealth 11, 464 (2014).

[9] W. Wang, Q. Liu, J. Liang, Y. Hu, and T. Zhou, Coevolution spreading in complex networks, Phys. Rep. 820, 1 (2019).

[10] N. F. Johnson, N. Velásquez, N. J. Restrepo, R. Leahy, N. Gabriel, S. El Oud, M. Zheng, P. Manrique, S. Wuchty, and Y. Lupu, The online competition between pro- and anti-vaccination views, Nature 582, 230 (2020).

[11] N. Oliver et al., Mobile phone data for informing public health actions across the COVID-19 pandemic life cycle, Sci. Adv. 6, eabc0764 (2020). 
[12] X. Wang, Y. Lan, and J. Xiao, Anomalous structure and dynamics in news diffusion among heterogeneous individuals, Nat. Human Behav. 3, 709 (2019).

[13] C. Granell, S. Gomez, and A. Arenas, Dynamical Interplay between Awareness and Epidemic Spreading in Multiplex Networks, Phys. Rev. Lett. 111, 128701 (2013).

[14] T. Gross, C. J. Dommar D'Lima, and B. Blasius, Epidemic Dynamics on an Adaptive Network, Phys. Rev. Lett. 96, 208701 (2006).

[15] F. Verelst, L. Willem, and P. Beutels, Behavioural change models for infectious disease transmission: a systematic review (2010-2015), J. R. Soc. Interface 13, 20160820 (2016).

[16] J. Hellewell, S. Abbott, A. Gimma, N. I. Bosse, C. I. Jarvis, T. W. Russell, J. D. Munday, A. J. Kucharski, W. J. Edmunds, Centre for the Mathematical Modelling of Infectious Diseases COVID-19 Working Group, S. Funk, and R. M. Eggo, Feasibility of controlling COVID-19 outbreaks by isolation of cases and contacts, Lancet Global Health 8, E488 (2020).

[17] R. M. Anderson, H. Heesterbeek, D. Klinkenberg, and T. D. Hollingsworth, How will country-based mitigation measures influence the course of the COVID-19 epidemic? Lancet $\mathbf{3 9 5}$, 931 (2020).

[18] S. Funk, E. Gilad, C. Watkins, and V. A. A. Jansen, The spread of awareness and its impact on epidemic outbreaks, Proc. Nat. Acad. Sci. (USA) 106, 6872 (2009).

[19] M. De Domenico, C. Granell, M. A. Porter, and A. Arenas, The physics of spreading processes in multilayer networks, Nat. Phys. 12, 901 (2016).

[20] G. F. De Arruda, F. A. Rodrigues, and Y. Moreno, Fundamentals of spreading processes in single and multilayer complex networks, Phys. Rep. 756, 1 (2018).

[21] Q. Guo, X. Jiang, Y. Lei, M. Li, Y. Ma, and Z. Zheng, Twostage effects of awareness cascade on epidemic spreading in multiplex networks. Phys. Rev. E 91, 012822 (2015).

[22] Z.-H. Lin, M. Feng, M. Tang, Z. Liu, C. Xu, P. M. Hui, and Y.-C. Lai, Non-Markovian recovery makes complex networks more resilient against large-scale failures, Nat. Commun. 11, 2490 (2020)
[23] Z. Wang, C. Xia, Z. Chen, and G. Chen, Epidemic propagation with positive and negative preventive information in multiplex networks, IEEE Trans. Cybernet.1 (2020).

[24] T. J. Philipson, Chapter 33 Economic epidemiology and infectious diseases, Handbook Health Econ. 1, 1761 (2000).

[25] L. Böttcher, O. Woolley-Meza, N. A. M. Araújo, H. J. Herrmann, and D. Helbing, Disease-induced resource constraints can trigger explosive epidemics, Sci. Rep. 5, 16571 (2015).

[26] A. K. Misra, R. K. Rai, and Y. Takeuchi, Modeling the effect of time delay in budget allocation to control an epidemic through awareness, Int. J. Biomath. 11, 1850027 (2018).

[27] H. Zhang, Z. Wu, M. Tang, and Y. Lai, Effects of behavioral response and vaccination policy on epidemic spreading-an approach based on evolutionary-game dynamics, Sci. Rep. 4, 5666 (2015).

[28] C. T. Bauch and D. J. D. Earn, Vaccination and the theory of games, Proc. Nat. Acad. Sci. (USA) 101, 13391 (2004).

[29] R. Schulz and P. R. Sherwood, Physical and mental health effects of family caregiving, Ame. J. Nursin. 108, 23 (2008).

[30] T. E. Seeman, Social ties and health: the benefits of social integration, Ann. Epidemiol. 6, 442 (1996).

[31] X. Chen, R. Wang, M. Tang, S. Cai, H. E. Stanley, and L. A. Braunstein, Suppressing epidemic spreading in multiplex networks with social-support, New J. Phys. 20, 013007 (2018).

[32] T. Aoki and T. Aoyagi, Scale-free Structures Emerging from Co-Evolution of a Network and the Distribution of a Diffusive Resource on It, Phys. Rev. Lett. 109, 208702 (2012).

[33] S. C. Ferreira, C. Castellano, and R. Pastor-Satorras, Epidemic thresholds of the susceptible-infected-susceptible model on networks: a comparison of numerical and theoretical results, Phys. Rev. E 86, 041125 (2012).

[34] M. Magnani, B. Micenkova, and L. Rossi, Combinatorial analysis of multiple networks, arXiv:1303.4986.

[35] R. Pastor-Satorras, C. Castellano, P. Van Mieghem, and A. Vespignani, Epidemic processes in complex networks, Rev. Mod. Phys. 87, 925 (2015). 